\title{
ABSCESO CERVICAL PROFUNDO CON RIESGO DE OBSTRUCCIÓN DE VÍA AÉREA EN UN LACTANTE. DESCRIPCIÓN DE UN CASO
}

\section{Deep neck abscess with risk of airway obstruction in an infant. A case report}

\author{
Eugenia LÓPEZ-SIMÓN; Patricia CORRIOLS-NOVAL; Nathalia CASTILLO-LEDESMA; Carmelo \\ MORALES-ANGULO \\ Servicio de Otorrinolaringología. Hospital Universitario Marqués de Valdecilla. Santander. España. \\ Correspondencia:eugenialopez@usal.es
}

Fecha de recepción: 17 de diciembre de 2018

Fecha de aceptación: 26 de enero de 2019

Fecha de publicación: 3 de febrero de 2019

Fecha de publicación del fascículo: 1 de diciembre de 2019

Conflicto de intereses: Los autores declaran no tener conflictos de intereses.

Imágenes: Los autores declaran haber obtenido las imágenes con el permiso de los pacientes.

Política de derechos y autoarchivo: se permite el autoarchivo de la versión post-print (SHERPA/RoMEO).

Licencia CC BY-NC-ND. Licencia Creative Commons Atribución-NoComercial-SinDerivar 4.0 Internacional.

Universidad de Salamanca. Su comercialización está sujeta al permiso del editor.

RESUMEN: Introducción y objetivo: Los abscesos retrofaríngeos son actualmente poco frecuentes en niños. Ocurren normalmente en menores de 5 años y son en su mayoría infecciones polimicrobianas. Método: Se presenta el caso de un niño de 6 meses que presentó un absceso de $7 \mathrm{~cm}^{3}$ de volumen que requirió incisión y drenaje por vía transoral. Se usó la lista de comprobación CARE para la redacción del caso. Los padres del paciente consintieron el uso de sus datos clínicos. Discusión: La tomografía axial computarizada es la prueba diagnóstica de elección. Se debe realizar siempre que se sospechen infecciones profundas del cuello. El tratamiento puede ser conservador en su inicio mediante terapia antibiótica intravenosa. Requerirán drenaje quirúrgico si empeoran clínicamente y en aquellos abscesos de gran tamaño con riesgo de obstrucción de la vía aérea. Conclusiones: Los abscesos cervicales profundos de gran tamaño en lactantes son una patología rara en la actualidad, pero potencialmente grave. Es importante realizar un diagnóstico y tratamiento precoz para evitar complicaciones serias.

Palabras clave: infecciones profundas del cuello; niños; absceso retrofaríngeo; absceso parafaríngeo; drenaje; tomografía computarizada. 


\title{
ABSCESO CERVICAL PROFUNDO CON RIESGO DE OBSTRUCCIÓN DE VÍA AÉREA EN UN LACTANTE. DESCRIPCIÓN DE UN CASO \\ LÓPEZ-SIMÓN E ET AL
}

\begin{abstract}
SUMMARY: Introduction and objective: Retropharyngeal abscess are uncommon. They usually occur in children younger than 5 years and most of them are polymicrobial infections. Method: We report a case of a six-month-old boy with an abscess of $7 \mathrm{~cm}^{3}$ that required incision and transoral surgical drainage. We used the CARE checklist when writing our report [8]. Parents of patient gave their consent to use clinical data for publication. Discussion: Computed tomography of the neck is the diagnostic technique of choice. It has to be done when deep neck infections are suspected. Conservative treatment with intravenous antibiotics would be the first choice. Prompt surgical drainage would be needed if symptoms worsen or in large abscesses with risk of airway obstruction. Conclusions: Deep neck abscesses are uncommon in infants at present, but potentially serious. An early diagnosis and treatment are important to avoid severe complications.

Keywords: deep neck infections; children; retropharyngeal abscess; parapharyngeal abscess; drainage; computed tomography.
\end{abstract}

\section{INTRODUCCIÓN}

Los abscesos retrofaríngeos son en la actualidad bastante raros y ocurren más frecuentemente en niños menores de 5 años que en adultos [1]. Aproximadamente, dos tercios de los pacientes tienen antecedentes de infección ótica, nasal o faríngea reciente [2]. Las manifestaciones clínicas son inespecíficas y consisten en fiebre, irritabilidad, reducción de la ingesta por vía oral y babeo. También se puede observar rigidez de cuello o tortícolis. El niño que ya habla podría referir dolor de garganta y de cuello. Otros signos son Voz apagada, estridor y dificultad respiratoria, e incluso apnea obstructiva del sueño [3]. La exploración física puede poner de manifiesto un abultamiento en la pared faríngea posterior, pero éste aparece en menos del 50\% de los lactantes. El diagnóstico diferencial lo integran la epiglotitis aguda y la aspiración de cuerpos extraños, principalmente. Aunque la ecografía cervical puede contribuir al diagnóstico de un absceso profundo cervical, la tomografía axial computarizada (TC) o la resonancia magnética (RM) es fundamental para conocer su extensión y evaluar la necesidad de realizar un drenaje quirúrgico del mismo [4]. Las opciones terapéuticas incluyen antibioterapia intravenosa asociada o no a drenaje quirúrgico [5, 6]. Se ha demostrado que más del $50 \%$ de los niños con un absceso retrofaríngeo se pueden tratar sin necesidad de drenaje quirúrgico $[2,6]$. Este drenaje será necesario en pacientes con abscesos mayores de $2 \mathrm{~cm}$, dificultad respiratoria o que no mejoren tras la antibioterapia intravenosa $[3,7]$. Los abscesos profundos en lactantes son poco frecuentes. Se presenta un caso clínico de un lactante de 6 meses de edad de gran tamaño y se comenta el manejo de los abscesos profundos en niños de corta edad con alto riesgo de obstrucción de vía aérea.

\section{DESCRIPCIÓN}

Para la descripción del caso se siguen las directrices CARE [8].

Información del paciente: Lactante de 6 meses que fue traído a urgencias remitido por su pediatra de referencia por un cuadro de 24 horas de evolución de fiebre $\left(38.2^{\circ} \mathrm{C}\right)$, llanto e irritabilidad. Como antecedentes personales conocidos del paciente destacaba una fibromatosis colli del esternocleidomastoideo derecho en seguimiento. La semana previa sufrió un cuadro de faringoamigdalitis por Streptococcus pyogenes. Hallazgos clínicos: En la exploración física se encontraba con estado general estable y manteniendo buenas constantes. La faringe se mostraba hiperémica, sin exudados vesículas ni petequias. Resto de la exploración sin hallazgos patológicos significativos. Evaluación diagnóstica: Se realizó una radiografía de tórax, analítica de sangre y orina que resultaron 


\section{ABSCESO CERVICAL PROFUNDO CON RIESGO DE OBSTRUCCIÓN DE VÍA AÉREA EN UN LACTANTE. DESCRIPCIÓN DE UN CASO \\ LÓPEZ-SIMÓN E ET AL}

ser normales por lo que se decidió observación en domicilio. Pasadas 24 horas, acudió de nuevo por empeoramiento clínico. Se repitieron las pruebas analíticas observando $34.9 \times 10^{3} / \mathrm{ml}$ leucocitos ( $56,3 \%$ segmentados) y proteína $\mathrm{C}$ reactiva de $12,5 \mathrm{mg} / \mathrm{dl}$. El cultivo de orina informaba crecimiento de $>100000$ UFC de E. coli. Por lo que, dado el rechazo a la alimentación y la afectación del estado general, se decidió ingreso hospitalario para administración de ceftriaxona intravenosa por sospecha de infección del tracto urinario. En las 24 horas siguientes, se observó la aparición de una tumoración laterocervical izquierda de consistencia blanda, sin eritema ni calor a la palpación, de $3.3 \mathrm{x}$ 2.7 x $3.4 \mathrm{~cm}$ sugestiva de absceso retrofaríngeo en la ecografía realizada de urgencia por lo que contactaron para valoración ORL. La exploración física llevada a cabo en consulta no demostró limitación a la movilización cervical ni trismus. Orofaringe difícil de visualizar, con abundante mucosidad en cavum, que no permite descartar abombamiento laterofaríngeo o posterior. Se realizó nasofibrolaringoscopia visualizando gran abombamiento laterofaríngeo izquierdo. En la TC de urgencia se confirmó la existencia de una masa parafaríngea izquierda de al menos $7 \mathrm{ml}$ de volumen, con una pared gruesa que captaba contraste y contenido homogéneo de menor densidad, que desplazaba y comprimía la vía aérea a ese nivel en íntima continuidad con la misma, compatible con linfadenitis supurativa retrofaríngea (Figura 1). Intervención terapéutica: Dado el tamaño de este, se decidió someter al paciente a drenaje quirúrgico transoral bajo anestesia general sin incidencias quirúrgicas ni anestésicas. Se llevó a cabo desbridamiento, limpieza con agua oxigenada y envío de muestras para cultivo que resultó ser positivo para Streptoccocus pyogenes. Seguimiento y resultados: La exploración física posterior no evidenció signos palpables de la masa. El paciente estuvo bajo observación en la Unidad de Cuidados Intensivos Pediátrica (UCIP) en tratamiento intravenoso con clindamicina y tobramicina donde permaneció intubado hasta mejoría clínica. El tercer día de postoperatorio, los resultados de la analítica mejoraron significativamente y tras la realización de nueva ecografía de control sin objetivar signos de recidiva del absceso, se decidió la extubación del paciente. Tras siete días en la UCIP con buena evolución clínica, se decidió traslado a la planta de pediatría para continuar con tratamiento intravenoso pautado y vigilancia. A la exploración, no se palpaban nuevas masas ni adenopatías, presentaba buena movilidad cervical, sin contracturas. La nasofibrolaringoscopia estaba dentro de la normalidad y las cifras analíticas volvieron a valores basales. Por lo tanto, tras diez días ingresado se decidió alta a domicilio para completar pauta de tratamiento antibiótico oral (clindamicina $300 \mathrm{mg}$ siete días). Tres meses después el paciente se encontraba con buen estado general y fue derivado al servicio de Inmunología para descartar una posible inmunodeficiencia primaria, que fue descartada finalmente.

\section{DISCUSIÓN}

Los abscesos retro y parafaríngeos son complicaciones infecciosas del espacio cervical profundo poco frecuentes actualmente y que suelen acontecer en niños menores de 5 años más que en adultos. Sin embargo, son muy infrecuentes en lactantes menores de 1 año [1]. La mitad de estos refieren antecedentes de infección de vías altas previa al episodio que provoca un cuadro de linfadenitis supurativa cervical y un menor porcentaje es atribuido a haber sufrido algún traumatismo en el espacio retrofaríngeo [2]. En algunos países, ha aumentado su incidencia, según se reflejan en publicaciones previas debido a la mayor disponibilidad de técnicas diagnósticas por imagen como la TC y la mayor virulencia de los gérmenes (Streptococcus betahemolítico del grupo A y Staphylococcus aureus meticilín resistente) [7]. Dichos gérmenes junto con anaerobios son los causantes principales de los abscesos cervicales profundos, aunque en su mayoría son polimicrobianos [2, 7]. El espacio 


\section{ABSCESO CERVICAL PROFUNDO CON RIESGO DE OBSTRUCCIÓN DE VÍA AÉREA EN UN LACTANTE. DESCRIPCIÓN DE UN CASO \\ LÓPEZ-SIMÓN E ET AL}

cervical profundo está formado por tres fascias y tres espacios donde tanto infecciones como hematomas pueden acontecer y comprometer la vía aérea. Los abscesos retrofaríngeos se manifiestan clínicamente en su comienzo de forma similar a una faringitis no complicada. En su forma más temprana, los enfermos se encuentran febriles y con eritema faríngeo leve-moderado.

A medida que la infección progresa, la inflamación se vuelve más prominente y son incluso incapaces de tolerar sus propias secreciones orales. Además, hay signos y síntomas que deben alertar por corresponder a la obstrucción de vía aérea o digestiva como disfagia, odinofagia, tortícolis, trismus, cambios en la voz, distrés respiratorio, etc. $[1,2,7]$. La exploración física del paciente con probable absceso cervical profundo debe ser realizada por especialistas experimentados en manejo de la vía aérea urgente pues la ruptura puede acontecer durante la misma. Se ha sugerido por ello, realizar la palpación cervical en posición de Trendelenburg y con todo el material disponible preparado para prevenir su aspiración y consecuente asfixia, sobre todo cuando se trata de abscesos de lactantes de corta edad como en nuestro caso [5]. El diagnóstico clínico se complementa con el análisis de laboratorio y las técnicas por imagen. Diferentes autores hallaron el recuento de leucocitos medio entre $20500-25000 / \mathrm{mm}^{3}$, con aumento de los reactantes de fase aguda [5]. La radiografía cervical en proyección lateral es la técnica de elección en la primera evaluación del absceso, sobre todo en niños pequeños. Debe ser obtenida en inspiración y con el cuello en extensión. Sin embargo, la TC con contraste es lo más sensible, aunque poco específico, y contribuye a conocer la localización exacta del mismo, su tamaño, su relación con las estructuras vasculares del cuello y la vía aérea, y ayuda a planificar la cirugía en caso de que fuese necesaria $[4,6,7]$. Los abscesos cervicales profundos deben diferenciarse de patologías más comunes, como la linfadenitis cervical y parotiditis, de tumoraciones cervicales (desde
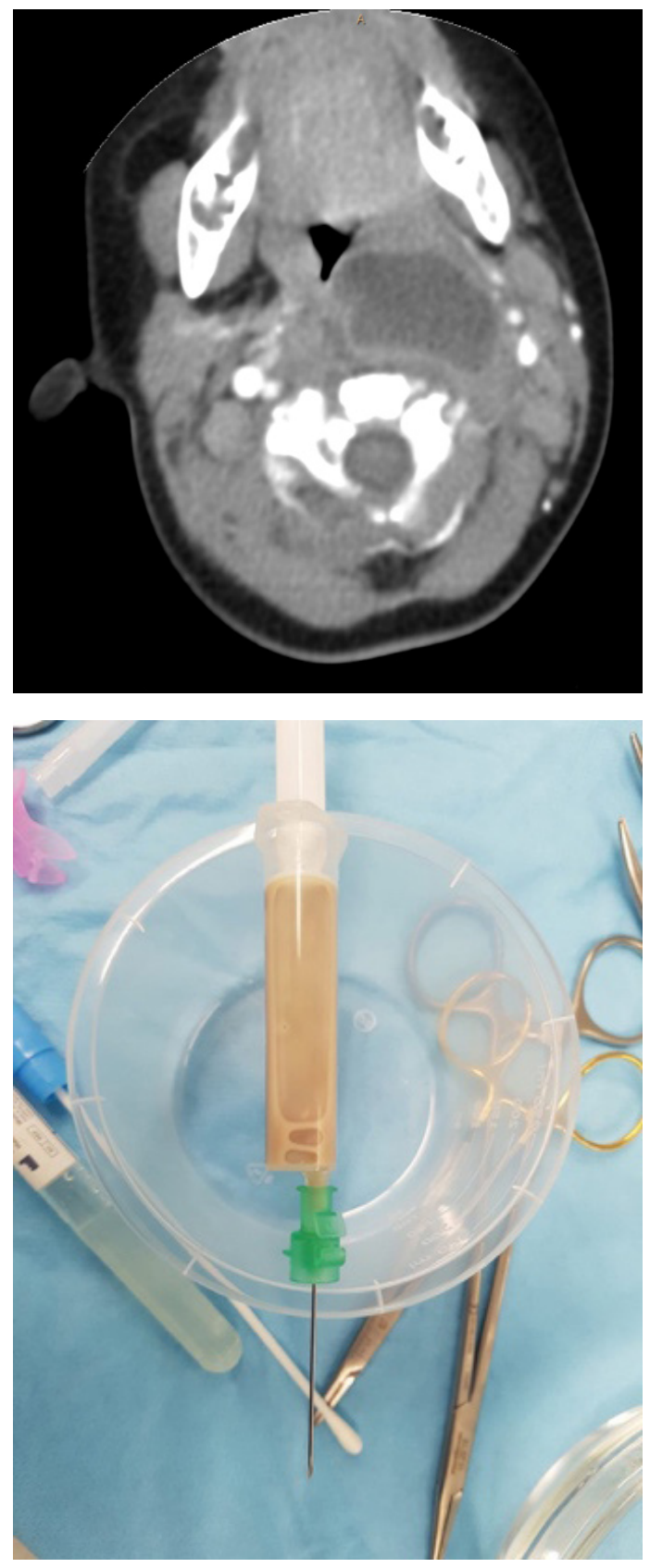

Figura 1. Absceso parafaríngeo izquierdo de aproximadamente $7 \mathrm{ml}$ de volumen y muestra obtenida mediante drenaje transoral bajo anestesia general. 


\section{ABSCESO CERVICAL PROFUNDO CON RIESGO DE OBSTRUCCIÓN DE VÍA AÉREA EN UN LACTANTE. DESCRIPCIÓN DE UN CASO \\ LÓPEZ-SIMÓN E ET AL}

quiste tirogloso infectado hasta linfoma). Aunque con los tratamientos actuales han disminuido de forma significativa las complicaciones secundarias, es importante un diagnóstico y tratamiento precoz para evitarlas [6]. Se han descrito lesiones de estructuras contiguas, obstrucción de la vía aérea, sepsis, mediastinitis, síndrome de Lemierre, entre otras [5]. Todos los pacientes con diagnóstico confirmado de infección retrofaríngea deben ser hospitalizados y tratados con antibióticos intravenosos que cubran gérmenes tanto aerobios como anaerobios. Aquellos que no manifiesten compromiso respiratorio, pueden ser tratados de forma conservadora y reevaluados tras 24 a 48 horas [4]. ¿Es necesario añadir corticoides sistémicos a dosis antiinflamatorias desde el inicio del cuadro? La utilización de una dosis alta de corticoides es controvertida. La indicación quirúrgica de los abscesos retro y parafaríngeos se reserva, de entrada, para pacientes con abscesos grandes que puedan poner en riesgo la vida del lactante o en casos de empeoramiento de la sintomatología clínica tras la instauración del tratamiento antibiótico [6]. La vía de acceso viene determinada por la localización del absceso en la TC, siendo de elección el acceso transoral cuando este es factible [2].

\section{CONCLUSIONES}

Los abscesos cervicales profundos de gran tamaño en lactantes son una patología rara en la actualidad, pero potencialmente grave. Es importante realizar un diagnóstico y tratamiento precoz para evitar complicaciones serias.

\section{BIBLIOGRAFÍA}

1. Brown NK, Hulten KG, Mason EO, Kaplan SL. Staphylococcus aureus retropharyngeal abscess in children. Pediatr. Infect. Dis. J. 2015;34(4):454-6.

2. Choche Santander B, Prieto del Prado A, Madrid Castillo MD, Neth O, Obando Santaella I. Abscesos retrofaríngeo y parafaríngeo: experiencia en hospital terciario de Sevilla durante la última década. An Pediatr (Barc) 2011;75(4):266-72.

3. Grisaru-Soen G, Komisar O, Aizenstein O, Soudack M, Schwartz D, Paret G. Retropharyngeal and parapharygeal abscess in children - epidemiology, clinical features and treatment. Int J Pediatr Otorhinolaryngol 2010;74(9):1016-20.

4. Woods CR, Cash ED, Smith AM, Smith MJ, Myers JA, Espinosa CM, et al. Retropharyngeal and Parapharyngeal Abscesses Among Children and Adolescents in the United States: Epidemiology and Management Trends. J Pediatric Infect Dis Soc. 2016 Sep;5(3):259-68.

5. Ramos Díaz JC, Rizo Hoyos M, Cañuelo Ruiz O, Trigo Moreno J, Fernández Gómez, E. Infecciones profundas del cuello: abscesos retro y parafaríngeos. An Pediatr (Barc) 2010;72(5):359-60.

6. Page NC, Bauer EM, Lieu JE. Clinical features and treatment of retropharyngeal abscess in children. Otolaryngol Head Neck Surg 2008;138(3):300-6. 2.

7. Novis S, Pritchett C, Thorne M, Gordon H. Pediatric eep space neck infections in U.S. children, 2000-2009. Int J Pediatr Otorhinolaryngol 2014;78(5): 832-6.

8. Gagnier JJ, Kienle G, Altman DG, Moher D, Sox H, Riley D; the CARE Group. The CARE Guidelines: Consensus-based Clinical Case Reporting Guideline Development. (http://www.goodreports.org/ care) 
